\title{
The efficacy and safety of different radiotherapy doses in neoadjuvant chemoradiotherapy for locally advanced rectal cancer
}

\author{
Yongchun Zhang, Peng Jiang, Hui Zhu, Bin Dong, Hanxiao Zhai, Zhiying Chen \\ Department of Radiation Oncology, The Affiliated Hospital of Qingdao University, Qingdao, China \\ Contributions: (I) Conception and design: Z Chen; (II) Administrative support: Y Zhang; (III) Provision of study materials or patients: P Jiang, H Zhu, \\ B Dong, H Zhai; (IV) Collection and assembly of data: All authors; (V) Data analysis and interpretation: All authors; (VI) Manuscript writing: All \\ authors; (VII) Final approval of manuscript: All authors. \\ Correspondence to: Yongchun Zhang; Zhiying Chen. Department of Radiation Oncology, The Affiliated Hospital of Qingdao University, No. 16 \\ Jiangsu Road, Shinan District, Qingdao 266000, China. Email: zyc18661805058@163.com; 18661804759@163.com.
}

Background: This study aimed to evaluate efficacy and adverse effects of different radiotherapy (RT) doses in neoadjuvant chemoradiotherapy for locally advanced rectal cancer.

Methods: Fifty-nine patients with locally advanced rectal cancer who underwent neoadjuvant chemoradiotherapy in hospital between January 2015 and May 2017 were enrolled in retrospective analysis. The patients were divided into the 56-Gy group and the 50-Gy group. The concurrent chemotherapy regimen was based on capecitabine. All patients received one cycle of oxaliplatin combined with capecitabine induction chemotherapy. All patients completed neoadjuvant chemoradiotherapy and received radical surgery.

Results: Of the patients in this study, 29 patients and 30 patients received a radiation dose of 56- and 50-Gy, respectively. All clinical characteristics were matched between the two groups. All patients received surgery 6 to 8 weeks after completing RT. The therapeutical effective rate in the 56-Gy group was $93.10 \%$ (27/29), compared with $66.67 \%$ in the 50-Gy group (20/30); the difference between the two groups was statistically significant $\left(\chi^{2}=6.36, \mathrm{P}=0.01\right)$. The pathological complete remission (pCR) rate in the 56-Gy group $(37.93 \%, 11 / 29)$ was statistically significantly higher than that in the 50-Gy group $(13.33 \%$, 4/30) $\left(\chi^{2}=4.71, P=0.030\right)$. The anal preservation rate in the $56-G y$ group $(65.5 \%, 19 / 29)$ was statistically significantly higher than that in the 50-Gy group $(33.33 \%, 10 / 30)\left(\chi^{2}=6.11, \mathrm{P}=0.01\right)$. The 56-Gy group had a local recurrence rate of $0 \%(0 / 29)$ and a distant metastasis rate of $10.34 \%(3 / 29)$, while the 50-Gy group had a local recurrence rate of $6.67 \%(2 / 30)$ and a distant metastasis rate of $16.67 \%(5 / 30)$; no significant difference existed between the two groups $\left(\chi^{2}=2.00,0.50, \mathrm{P}=0.16,0.48\right)$. The incidence of adverse reactions (gastrointestinal reactions, bone marrow suppression, and perianal skin reactions) in the 56-Gy group was not significantly different from that in the 50-Gy group $(\mathrm{P}>0.05)$.

Conclusions: Increasing the radiation dose can significantly improve the anal preservation and pCR rates of patients with locally advanced rectal cancer, thus improving their life quality. Moreover, it does not increase the rates of recurrence or adverse reactions. Our findings have certain clinical significance, but further prospective study is needed.

Keywords: Locally advanced rectal cancer; concurrent chemoradiotherapy; dose segmentation; curative effect; chemoradiotherapy side effects

Submitted Apr 29, 2021. Accepted for publication Jul 13, 2021.

doi: 10.21037 /jgo-21-296

View this article at: https://dx.doi.org/10.21037/jgo-21-296 


\section{Introduction}

Rectal cancer is one of the most common gastrointestinal cancers. Owing to the lack of specific symptoms at the early stage, $70-80 \%$ of cases of rectal cancer are locally advanced at the time of clinical diagnosis, resulting in a poor treatment effect. Due to the improvement of surgical techniques, the extensive application of total mesorectal excision (TME), and the combined use of multiple treatment modes, the local control rate of rectal cancer has shown significant improvement in recent years (1-3). Neoadjuvant concurrent chemoradiation (nCRT) can significantly reduce the tumor burden of locally advanced rectal cancer, increase the rates of complete resection of local tumors and anal preservation, and greatly improve the local control rate of locally advanced rectal cancer (4). Furthermore, in locally advanced rectal cancer, nCRT can lead to significant downgrading of the primary lesion. In their clinical study, Sanghera et al. found that $42 \%$ of patients achieved pCR (5). However, the overall survival (OS) of rectal cancer has only improved slightly, mainly due to metastasis to distant organs, such as the liver and lung, occurring during the course of diagnosis and treatment (6-8).

The main factors affecting the prognosis of rectal cancer are tumor-node-metastasis (TNM) stage, lymph node metastasis, vascular invasion, and the peripheral margin. The surgical marginal margin and the extent of lymph node dissection also impact patient prognosis in rectal cancer. Following nCRT, the degree of tumor decline is another important prognostic factor.

At present, nCRT combined with TME is the standard treatment method for locally advanced rectal cancer, especially for low and middle rectal cancer (9-12). The National Comprehensive Cancer Network (NCCN) recommends nCRT as the preferred treatment option for low and middle rectal cancer. Patients with stage II (T3-4, lymph node-negative, and tumor invasion of the muscularis) and stage III (lymph node-positive) rectal cancer should be administered neoadjuvant radiotherapy (RT) at a dose of 45 to 50 Gy in 25 to 28 fractions before surgery. Surgery is performed 6-10 weeks after the completion of RT. To explore whether increasing the dose of local RT can further reduce tumor staging, improve the anal preservation and local control rates, and reduce the rate of distant metastases in rectal cancer, we retrospectively analyzed the clinical data of patients with locally advanced disease who received conventional-dose or high-dose RT between January 2015 and May 2017, all patients are Asian. The patients were divided into the 50-Gy group and the 56-Gy group, and differences in treatment efficacy and safety between the two groups were compared. We present the following article in accordance with the STROBE reporting checklist (available at https://dx.doi.org/10.21037/jgo-21-296).

\section{Methods}

\section{Inclusion criteria}

The inclusion criteria for patients were: (I) a diagnosis of rectal adenocarcinoma, as determined by colonoscopy; (II) lower edge of the tumor within $12 \mathrm{~cm}$ of the anal edge; (III) a locally advanced clinical stage (i.e., T3-T4 or $\mathrm{N+}$ ), as determined by pelvic magnetic resonance imaging (MRI) or ultrasound endoscopy (Figure 1); (IV) underwent thoracoabdominal computed tomography (CT) or positron emission tomography (PET)/CT to exclude distant metastases; (V) general condition score of 0 or 1; (VI) good bone marrow hematopoiesis, and liver, kidney, and heart function; (VII) underwent complete preoperative nCRT and radical rectal cancer surgery and had complete surgical data. This research was approved by the local ethics committee of Affiliated Hospital of Qingdao University. The study was conducted in accordance with the Declaration of Helsinki (as revised in 2013). All participants in the study gave informed consent to this study and signed an informed consent form.

\section{Exclusion criteria}

The exclusion criteria for patients were: (I) underwent thoracoabdominal CT or PET/CT, clinically diagnosed as an advanced patient with distant organ metastasis; (II) previous history of chemoradiotherapy or targeted therapy.

\section{Treatment programs}

\section{RT plan}

All patients were fixed in the prone position, then accept enhanced CT scan to positioning (scan layer thickness is $5 \mathrm{~mm}$ ), and maintain bladder filling during positioning and RT. Intensity-modulated RT (IMRT) was performed using the Eclipse 13.6 version planning system. The target area was delineated according to the North American Radiation Therapy Oncology Group (RTOG) standard.

In the 56-Gy group: the gross tumor volume (GTV-t) was defined as the primary tumor detected by physical and imaging examinations; the metastatic lymph node volume 

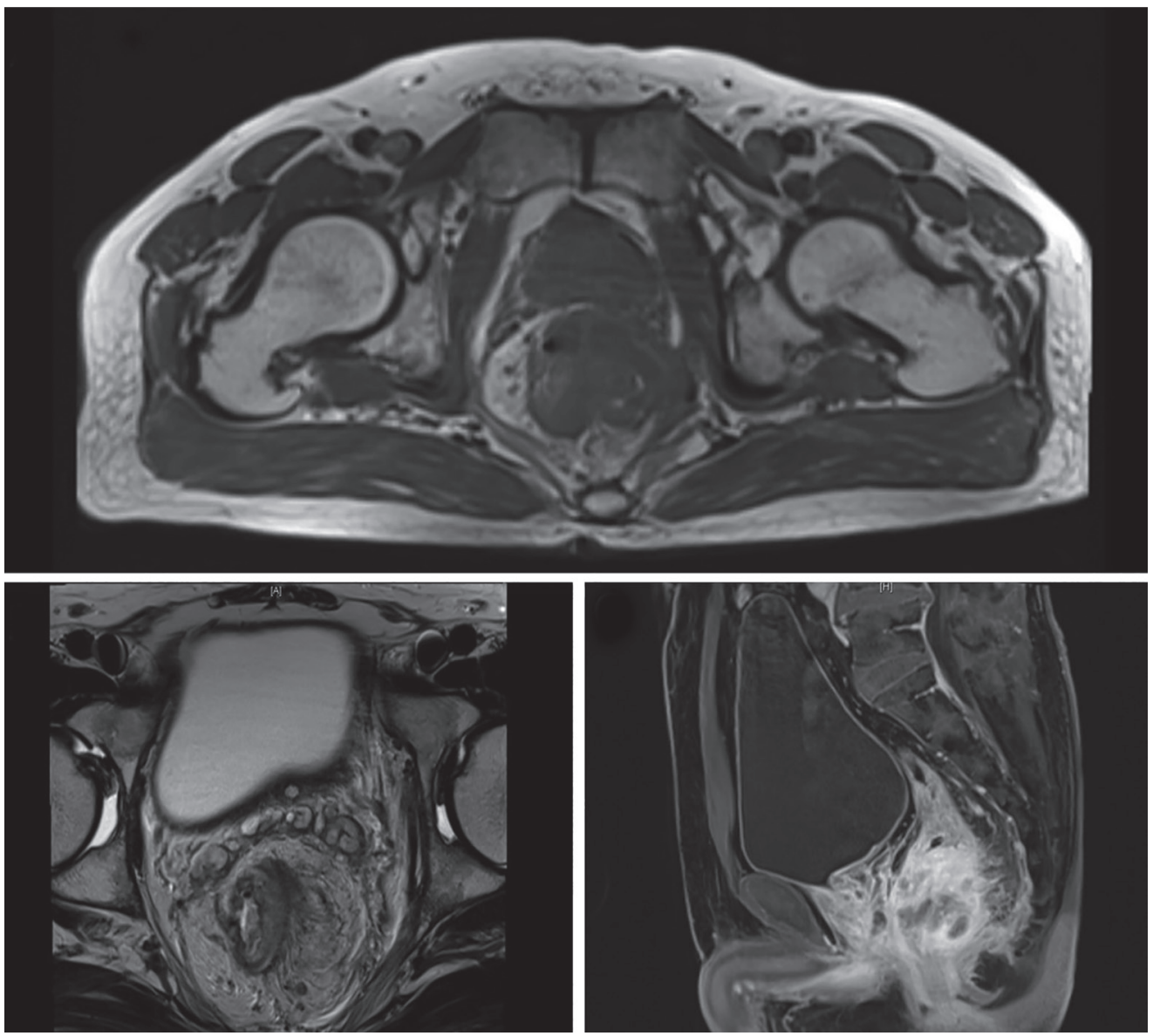

Figure 1 Rectal high-resolution MRI of a representative patient. The distance from the lower edge of the tumor to the anal edge is $21 \mathrm{~mm}$; the distance from the puborectalis/anorectal angle is $0 \mathrm{~mm}$; and the range of the upper and lower edges of the tumor is $75 \mathrm{~mm}$; the main body of the tumor are located on the intestinal wall: T4b; the local boundary with the left seminal vesicle gland is unclear and is located in the direction of 0-6 points. Anal complex evaluation: stage A4, the left anal canal and puborectalis muscle space are not visible, and the left puborectalis muscle has thickened. Lymph node evaluation: N2, more than four long T2 nodes with abnormal signals, the larger one measures about $10 \mathrm{~mm}$. Evaluation of perirectal circumcision: CRM (+). Evaluation of extramuscular vascular invasion EMVI: EMVI (+). Rectal cancer preoperative assessment: low, T4bN2Mx, CRM (+), EMVI (+). MRI, magnetic resonance imaging; CRM, circumferential resection margin; EMVI, extramural venous invasion.

(GTV-n) was defined as pelvic metastatic lymph nodes; and the clinical high-risk target volume (CTV-hr) included the GTV-t and the entire rectum, mesentery, and presacral region, as well as all visible mesentery lymph nodes. The scope of the clinical low-risk target volume (CTV-lr) was defined as follows. For stage T3 tumors, it included the complete mesenteric, and left and right iliac lymphatic drainage areas. For stage T4 tumors with prerectal organ invasion, it included the left and right iliac external lymphatic drainage areas and a $1-2-\mathrm{cm}$ area around the tumor in the invading adjacent organs. The upper boundary included the entire rectum and mesentery (usually located at L5/S1) and an area of at least $2 \mathrm{~cm}$ above the rectal lesion, and the lower pelvic floor or an area of at least $2 \mathrm{~cm}$ below the rectal lesion. For the lymphatic drainage area, it included a $0.7-\mathrm{cm}$ border outside the iliac vessels. For the extra-iliac lymph drainage area, it included the borders of the extra iliac vessels and the sides plus $1 \mathrm{~cm}$. The anterior border took into account the changes in the filling status of the bladder and rectum, and included a bladder range of $1-1.5 \mathrm{~cm}$. It was necessary to include the obturator lymph node area between the inner and outer iliac vessels. 

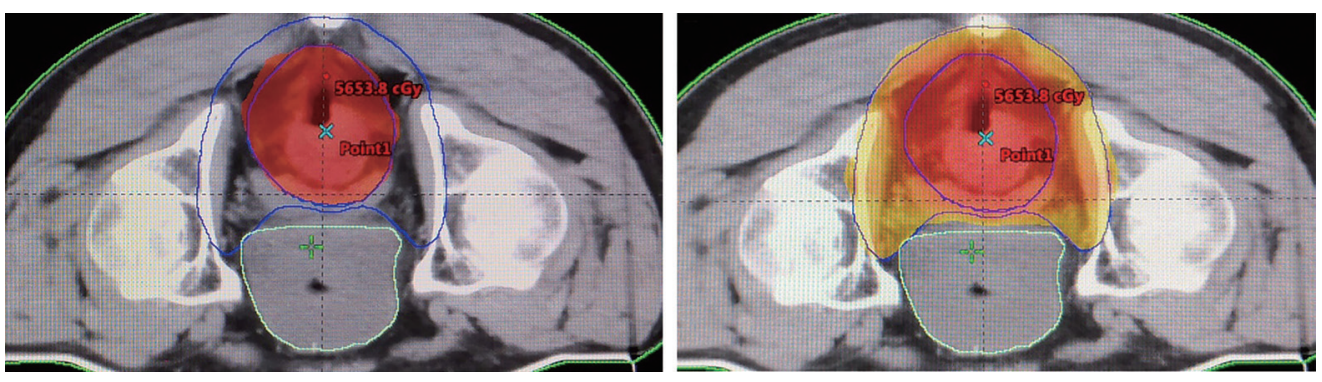

Figure 2 The 56-Gy group. Radiation target dose distribution, PTV-hr includes the entire rectum, mesentery, and presacral region within its scope, as well as all visible mesentery lymph nodes. The prescription dose is 56 Gy/27 f. The PTV-lr includes the complete mesenteric, and the left and right iliac lymphatic drainage areas. The upper boundary includes the entire rectum and mesentery (usually located at $\mathrm{L} 5 / \mathrm{S} 1$ ) or an area at least $2 \mathrm{~cm}$ above the rectal lesion, and the lower pelvic floor or a border at least $2 \mathrm{~cm}$ below the rectal lesion. For the lymphatic drainage area, RT target should include a $0.7-\mathrm{cm}$ border outside the iliac vessels. It is necessary to include the obturator lymph node area between the inner and outer iliac vessels. The prescription dose is 46 Gy/27 f. PTV-hr, planned high-risk target volume; PTV-lr, planned low-risk target volume; RT, radiotherapy.

In the 50-Gy group: the GTV-t was defined as the primary tumor found by physical and imaging examinations. The scope of the clinical target volume (CTV) was defined as follows. For stage T3 tumors, it included the entire rectal mesentery, and left and right sacral lymphatic drainage areas. For stage T4 tumors with prerectal organ invasion, it included the left and right external sacral lymphatic drainage areas and a $1-2-\mathrm{cm}$ area outside the tumor in the invading adjacent organs. The upper boundary included the entire rectum and mesentery (usually located at L5/S1) and an area of at least $2 \mathrm{~cm}$ above the rectal lesion, and the lower pelvic floor or an area of at least $2 \mathrm{~cm}$ below the rectal lesion. For the lymphatic drainage area, it included a $0.7-\mathrm{cm}$ border outside the iliac vessels. For the extrailiac lymph drainage area, it included the borders of the extra iliac vessels and the sides plus $1 \mathrm{~cm}$. The anterior border took into account the changes in the filling status of the bladder and rectum and included a bladder range of $1-1.5 \mathrm{~cm}$. It was necessary to include the obturator lymph node area between the inner and outer iliac vessels.

IMRT was delivered to all patients via $6 \mathrm{MV}$-X-rays. The planned target volume (PTV) of the left and right and front and rear directions was CTV $+0.8 \mathrm{~cm}$; the PTV of the head and foot directions was $\mathrm{CTV}+1 \mathrm{~cm}$; and the metastatic lymph node target volume (PGTV-n) was GTV-n $+1 \mathrm{~cm}$. The patients were divided into two groups on the basis of the different RT doses they received. Of the patients, 29 were prescribed PGTV-n 60 Gy, 220 cGy/f, PCTV-hr 5556 Gy, 200-210 cGy/f, PCTV-lr 46-47 Gy, 170-180 cGy/f, 27 times (Figure 2), and 30 patients were prescribed PCTV
$50 \mathrm{~Gy}, 200 \mathrm{cGy} / \mathrm{f}, 25$ times. The prescribed doses covered at least $95 \%$ of PTV. Organs at risk (OAR) included the small intestine, bladder, femoral head, horsetail, and spinal cord, the dose limits for which were as follows: small intestine Dmax $<50$ Gy, bladder V50 $<10 \%$, femoral head Dmax $<45$ Gy, horsetail Dmax $<52$ Gy, and spinal cord Dmax $<45$ Gy.

\section{Chemotherapy protocol and surgical method}

Oxaliplatin combined with capecitabine was given for one cycle before RT, and single-agent capecitabine was given during RT. The combination chemotherapy regimen was oxaliplatin $130 \mathrm{mg} / \mathrm{m}^{2}$ and capecitabine $1,000 \mathrm{mg} / \mathrm{m}^{2}$, taken orally, twice a day for 14 days, every 3 weeks. Capecitabine was given as a single dose of $825 \mathrm{mg} / \mathrm{m}^{2}$, taken orally, twice a day for 14 days, and was stopped for 7 days. A comprehensive preoperative assessment, including pelvic MRI, chest and abdominal CT, was performed 6 to 8 weeks after nCRT. The surgical method was determined by the surgeon according to the specific situation.

We enrolled 59 patients with locally advanced rectal cancer who were treated in our hospital between January 2015 and May 2017, and divided them into the 56-Gy group and the 50-Gy group according to the dose of neoadjuvant RT.

\section{Evaluation of adverse reactions}

While receiving RT and chemotherapy, all patients were examined weekly through routine blood, and liver and 
kidney function tests. Treatment-related adverse events were evaluated according to the Common Terminology Criteria for Adverse Events (CTCAE) version 3.0, and the RTOG foundation.

\section{Observation indicators}

Postoperatively, pathologic response was evaluated by TRG, Dworak's standard. Grade 0 was defined as no tumor regression; grade 1 was defined as an obvious tumor mass with fibrous tissue hyperplasia or vascular disease; grade 2 was defined as obvious fibrosis with a small number of tumor cells; grade 3 was defined as an extremely small number of tumor cells in a large amount of fibrous tissue, with or without mucus components; and grade 4 was defined as complete remission of pathology, with no tumor cells and only fibrous tissue. Grade 4 is equal to pCR, and grade $2+3+4$ means therapy has effect.

\section{Statistical analysis}

Statistical analyses were performed with IBM SPSS 23.0 software (New York, NY, USA). The $\chi^{2}$ test was used to compare age, sex, stage, tumor site, pathological response rate, and $\mathrm{T}$ degradation rate between the two groups. $\mathrm{P}<0.05$ was considered to be statistically significant.

\section{Results}

\section{General information}

A total of 59 patients, aged 37 to 89 years, were enrolled. The average age of the 56 - and 50 -Gy group was $58 \pm 10.13$ and $55 \pm 7.56$, respectively. Of the patients, 42 were male and 17 were female. There were 34 patients with stage T3 tumors and 25 patients with stage T4 tumors; 20 patients were stage $\mathrm{N} 0,23$ were stage $\mathrm{N} 1$, and 16 were stage $\mathrm{N} 2$. Low, middle, and upper rectal cancers accounted for 22, 32, and 5 cases, respectively. Sphincter-preserving surgery was performed in 29 cases, and none in 30 cases (Table 1).

\section{Remission rate of patients in two groups}

In the 56-Gy group, 11 cases achieved a pCR, which translated to a pCR rate of $37.93 \%$. In the $50-$ Gy group, 4 cases achieved a pCR, and the pCR rate was $13.33 \%$. The therapeutical effective rate in the 56-Gy group was $93.10 \%$ (27/29), compared with $66.67 \%$ in the $50-$ Gy group (20/30); the difference between the two groups was statistically significant $\left(\chi^{2}=6.36, \mathrm{P}=0.01\right)$. And the $\mathrm{pCR}$ rate was statistically significantly higher in the 56-Gy group than in the 50-Gy group $\left(\chi^{2}=4.71, \mathrm{P}=0.030, \mathrm{P}<0.05\right.$, Table 2$)$.

\section{Comparison of curative effect and adverse events between the two groups}

\section{Curative effect}

In the 56-Gy group, which received high-dose RT, the anal preservation rate was $65.50 \%(19 / 29)$. In the 50-Gy group, which received conventional-dose RT, the anal preservation rate was $33.33 \%(10 / 30)$. Therefore, the anal preservation rate in the 56-Gy group was statistically significantly higher than that in 50-Gy group $\left(\chi^{2}=6.11, \mathrm{P}=0.01\right)$. In the $56-\mathrm{Gy}$ group, the local recurrence rate was $0 \%(0 / 29)$ and the distant metastasis rate was $10.34 \%(3 / 29)$, compared with rates of $6.67 \%(2 / 30)$ and $16.67 \%(5 / 30)$, respectively, in the 50-Gy group. No significant difference existed between the two groups $\left(\chi^{2}=2.00,0.50, \mathrm{P}=0.16,0.48\right.$, Table 3).

\section{Adverse events}

There was no significant difference in the toxicity of RT and chemotherapy (including the rate of adverse events such as gastrointestinal reaction, bone marrow suppression, and perianal skin reaction) between the 56-Gy (high-dose RT) group and the 50-Gy (conventional-dose RT) group ( $\mathrm{P}>0.05$, Table 4).

\section{Discussion}

With the improvement of people's economic level and the Westernization of lifestyles in recent years, high-fat, highprotein, and low-fiber diets have become more popular, which has led to yearly increases in the incidence of colorectal cancer. Colorectal cancer is a common malignant tumor of the digestive tract and is surpassed only by gastric cancer. Most cases of rectal cancer are locally advanced at first diagnosis (13). There is a contradiction in the treatment of simple operation, (I) a large range of resection (include anus) to cut clean but seriously affect patients' quality of lives; or (II) preserve the anus but cannot achieve the complete removal of the tumor, then patients have a high local recurrence rate. The high risk of local recurrence has promoted the development of multiple treatment methods $(6,14)$. For patients with locally advanced rectal cancer, nCRT has become the standard treatment mode and has 
Table 1 Patient and treatment characteristics

\begin{tabular}{|c|c|c|c|c|}
\hline Characteristics & 56-Gy group $(\mathrm{n}=29)$ & 50-Gy group $(n=30)$ & $\mathrm{P}$ & $\chi^{2} / \mathrm{t}$ \\
\hline Median & $58 \pm 10.13$ & $55 \pm 7.56$ & & \\
\hline Range & $37-89$ & $38-71$ & & \\
\hline Sex, $n / \%$ & & & 0.84 & 0.04 \\
\hline Female & $8 / 37.59$ & $9 / 30.00$ & & \\
\hline Clinical stagey, $n / \%$ & & & 0.97 & 0.88 \\
\hline T3NO & $6 / 20.69$ & $7 / 23.33$ & & \\
\hline T3N1 & $7 / 24.14$ & $5 / 16.67$ & & \\
\hline $\mathrm{T} 4 \mathrm{~N} 1$ & $5 / 17.24$ & $6 / 20.00$ & & \\
\hline T4N2 & $3 / 10.34$ & $4 / 13.33$ & & \\
\hline Distance from anal verge $(\mathrm{cm}), \mathrm{n}$ & & & 0.25 & 1.16 \\
\hline Median & $6.65 \pm 2.03$ & $5.99 \pm 2.12$ & & \\
\hline Low (0-5) & 7 & 15 & & \\
\hline Mid $(>5-10)$ & 18 & 14 & & \\
\hline High $(>10-15)$ & 4 & 1 & & \\
\hline Tumor differentiation, $\mathrm{n} / \%$ & & & 0.59 & 1.06 \\
\hline Non-sphincter-preserving & $10 / 34.50$ & $20 / 66.67$ & & \\
\hline
\end{tabular}

Age and distances from lower edge of tumors to anus were compared by independent-samples $t$-test. The comparisons of the other indicators are based on the Chi-square test. There was no significant difference $(P>0.05)$.

significantly improved local control rates. Distant metastasis is now the main cause of treatment failure in these patients.

A large number of patients with locally advanced rectal cancer can benefit from nCRT $(15,16)$. This treatment can reduce the tumor stage, and can also improve the anal preservation rate; furthermore, some patients who receive nCRT achieve clinical remission (CR) (17-19). The clinical trials FFCD92-03 and EORTC 22921 compared the effects of preoperative nCRT and preoperative RT alone. The results showed that for patients with resectable locally advanced rectal cancer, simultaneous preoperative nCRT greatly improved the pCR and local control rates; however, it failed to improve the rates of anal preservation and longterm survival (20).

At present, the European Society for Medical Oncology (ESMO) guidelines recommend two preoperative neoadjuvant RT methods: combinations of short-term fast fractionated RT with 5-fluorouracil and conventional fractionated RT with 5-fluorouracil. Nordic countries (such as Norway and Sweden) often use short-term fast large- 
Table 2 Results of postoperative pathological examination

\begin{tabular}{|c|c|c|c|c|}
\hline Characteristics & 56-Gy group $(n=29)$ & $50-$ Gy group $(n=30)$ & $\chi^{2}$ & $\mathrm{P}$ \\
\hline урт0 & $11 / 37.93$ & 4/13.33 & & \\
\hline ypT1 & $6 / 20.69$ & $5 / 16.67$ & & \\
\hline урт2 & $6 / 20.69$ & $2 / 6.67$ & & \\
\hline урT4 & $2 / 6.90$ & $10 / 33.33$ & & \\
\hline ypN stage, n/\% & & & 9.55 & 0.008 \\
\hline ypNO & $21 / 72.41$ & $18 / 60.00$ & & \\
\hline ypN1 & $8 / 27.58$ & $4 / 13.33$ & & \\
\hline Grade 4 (pCR) & $11 / 37.93$ & $4 / 13.33$ & 4.71 & 0.030 \\
\hline Grade 3 & $10 / 34.48$ & $7 / 23.33$ & & \\
\hline Grade 2 & $6 / 20.69$ & $9 / 30.00$ & & \\
\hline Grade 1 & $0 / 0$ & $8 / 26.66$ & & \\
\hline Grade 0 & $2 / 6.90$ & $2 / 6.67$ & & \\
\hline
\end{tabular}

$\mathrm{pCR}$, pathological complete remission.

Table 3 Curative effect after neoadjuvant therapy (n/\%)

\begin{tabular}{lccc}
\hline Group & $\begin{array}{c}\text { Anus-preserving } \\
\text { rate }\end{array}$ & $\begin{array}{c}\text { Local } \\
\text { recurrence rate }\end{array}$ & $\begin{array}{c}\text { Distant } \\
\text { metastasis rate }\end{array}$ \\
\hline $56-$ Gy & $19 / 65.50$ & $0 / 0$ & $3 / 10.34$ \\
$50-$ Gy & $10 / 33.33$ & $2 / 6.67$ & $5 / 16.67$ \\
$\chi^{2}$ & 6.11 & 2.00 & 0.50 \\
$\mathrm{P}$ & 0.01 & 0.16 & 0.48 \\
\hline
\end{tabular}

segment RT, which is associated with the advantage of quick completion of perioperative treatment. However, this approach also has many shortcomings due to the operation being performed 1 week after the end of RT. For instance: the tumor shrinkage is not obvious; the clinical decline rate is not sufficient; the positive rate of the incision margin is not reduced; and the anal preservation rate is not increase. Swedish studies have found that high single-dose RT (25 Gy/5 f) produces more acute and late radiation-related side effects, as well as higher rates of neuroradiological damage and postoperative complications (21). Further, short-term rapid and large-fractionated RT has a shorter treatment time than the normal RT and does not work well with the adequate doses of chemotherapeutic drugs. At present, the commonly used dose of RT is 50 Gy in total, administered during a 5-week period, with 2 Gy per fraction. The advantage of this method is that it involves sufficient recovery time for acute radiation reactions andtumor shrinkage time.

In this study, we aimed to determine whether increasing the dose of RT can increase the rates of pathological remission and anal preservation. In our study, 29 patients in the 56-Gy group were treated with IMRT using a $6 \mathrm{MV}$ $\mathrm{X}$-ray linear accelerator and received a dose of $56 \mathrm{~Gy}$. The anal preservation rate in the 56-Gy group was significantly higher than that in the 50-Gy group. However, no significant differences were found in the rates of local recurrence or distant metastasis, or the incidence of toxicities and side effects between the two groups. Thus, high-dose neoadjuvant RT improved the anal preservation rate among patients without increasing the incidence of postoperative complications or adverse reactions.

In previous clinical studies, capecitabine was used as a basic chemotherapeutic drug for nCRT in patients 
Table 4 Toxic effects of RT and chemotherapy in 56-Gy group and 50-Gy group (n/\%)

\begin{tabular}{|c|c|c|c|c|c|c|c|}
\hline Group & \multicolumn{3}{|c|}{ Gastrointestinal reaction } & \multicolumn{3}{|c|}{ Myelosuppression } & $\begin{array}{c}\text { Perianal skin } \\
\text { reaction }\end{array}$ \\
\hline$A(n=29)$ & $3 / 10.34$ & $2 / 6.70$ & $5 / 17.24$ & $10 / 59.45$ & $10 / 59.45$ & $5 / 17.24$ & $2 / 6.70$ \\
\hline$B(n=30)$ & $2 / 6.67$ & $2 / 6.67$ & $3 / 10.00$ & $9 / 30.00$ & $9 / 30.00$ & $6 / 20.00$ & $2 / 6.67$ \\
\hline$\chi^{2}$ & 0.26 & 0.00 & 0.66 & 0.14 & 0.14 & 0.07 & 0.00 \\
\hline
\end{tabular}

$\mathrm{RT}$, radiotherapy.

with locally advanced rectal cancer, and its efficacy and safety have been confirmed. In 2009, the NCCN also recommended capecitabine as an RT sensitizer for preoperative nCRT for rectal cancer (22). At the 2011 American Society of Clinical Oncology (ASCO) meeting, it was reported that the National Surgical Adjuvant Breast and Bowel Project R-04 (NASBP R-04) trial had found no significant difference in the rates of $\mathrm{pCR}$ and adverse reactions between capecitabine and 5 -fluorouracil are used in nCRT for rectal cancer. In a number of clinical studies, including STAR-01, ACCORD12/0405, NSABPR-04, and PETACC 6, it was found that oxaliplatin combined with capecitabine or 5-fluorouracil did not increase the pCR or degradation rate among patients with locally advanced rectal cancer; however, the adverse reactions were greatly improved (16,23-25). Therefore, the NCCN recommends the use of capecitabine or fluorouracil as an option for concurrent chemoradiotherapy. In this study, all patients were given oxaliplatin combined with capecitabine for one cycle of induction chemotherapy before RT to reach tumor decline, and it was hoped that relative control of subclinical metastases would be achieved before RT. After the completion of induction chemotherapy, concurrent chemoradiotherapy was given, and capecitabine was given as a single-agent sensitization treatment during the same period. The local control and distant metastasis rates of all patients were similar to those in previous studies.

In this clinical study, all of the patients' operations were completed 6 to 8 weeks after nCRT. Currently, there is controversy over the time of surgery after chemoradiotherapy. A French randomized trial compared tumor regression at different time intervals between 2 and 6 weeks after nCRT and found that patients with a 6-week interval to surgery had significant tumor regression after nCRT (26). Based on this study, 6 weeks after the completion of nCRT was selected as the optimal time point for surgery. Furthermore, retrospective studies have found that a prolonged interval before radical surgery after the completion of nCRT makes patients with rectal cancer more likely to attain pCR (27-30). However, a prolonged surgical after RT interval may cause tissue fibrosis, which makes the operation more challenging. In the GRECCAR-6 trial (31), patients were randomized for surgery at 7 or 11 weeks after nCRT. Although the pCR rate was similar between the two groups, the risk of surgical resection was significantly elevated for patients with an 11-week interval. At the same time, tumors have different levels of sensitivity to chemoradiotherapy. For chemoradiosensitive tumors, satisfactory pathological degradation may be obtained 6 to 8 weeks after nCRT. However, tumors resistant to nCRT may cause distant metastases with a prolonged surgical interval, thus threatening patients' survival (32). Therefore, at present, large-scale prospective clinical studies are needed to determine the interval between nCRT completion and radical rectal cancer surgery that can obtain the best surgical resection and tumor regression rates, and reduce the surgical risk.

At present, patients with locally advanced rectal cancer who have undergone nCRT and have been evaluated as complete CR (cCR) may consider non-surgical treatment (33-39); this approach is called the "watch-and-wait" (WW) strategy. However, close follow-up and review are needed for timely detection of recurrence and metastasis. Early recurrence and metastasis can be detected through review of serum tumor markers, colonoscopy, and high-resolution rectal MRI. Most local recurrences occur within 1 year after nCRT. The appearance of microscopic lesions in the primary tumor bed that reached cCR after nCRT is called "early relapse" or "early regeneration" (40,41). After salvage surgery for this group of patients, complete resection can still be obtained and the anus can be retained (40). Based on the above reasons, for patients with locally advanced 
rectal cancer who achieve clinical PR (cPR) after nCRT, the surgical interval can be appropriately extended to improve the surgical resection rate and tumor degradation. In a prospective clinical study $(42,43)$, patients with $\mathrm{T} 2$ and $\mathrm{T} 3$ locally advanced rectal cancer were enrolled and treated with nCRT and given a rectal brachytherapy dose (total 65 Gy). No surgical treatment was performed after that, and the 2-year cCR rate reached 58\% (34). However, large-scale prospective studies are required to confirm the effect and survival between the WW strategy and radical surgery (44). In the present study, the 56-Gy group had a significantly different cCR rate than the 50-Gy group. We propose that the cCR rate can be further increased by increasing the dose of local RT delivered to tumor lesions. The WW policy can be adopted to avoid surgical trauma and obtain similar or better survival benefits for suitable patients.

Similar to previous studies, the 56- and 50-Gy groups in this study showed no significant difference in the rates of distant metastasis, which is the main factor affecting the prognosis of patients with rectal cancer. The concept of total neoadjuvant therapy (TNT) has been proposed, and its theoretical advantage lies in improving the compliance of patients with full-dose and full-course chemotherapy. The TNT strategy eliminates systemic metastases through the systemic administration of high-dose and full-course chemotherapy. Past research has found that the average distant metastasis rate is $19-31 \%$. Another potential advantage of TNT is that early chemotherapy may increase both tumor regression and the pathological response rate. The pCR rate is associated with long-term disease-free survival $(45,46)$. Multiple chemotherapy regimens have been shown to greatly reduce the progress of rectal cancer metastatic lesions. However, further prospective research is needed to identify chemotherapeutic options for TNT that can further reduce distant metastasis of rectal cancer. In the EORTC 2292126 study (4), patients with rectal cancer showed poor tolerance to chemotherapy after surgery. Therefore, TNT may encourage better chemotherapy compliance in patients with locally advanced rectal cancer. Finally, tumor degradation may persuade some patients to opt for non-surgical treatment or retain sphincter surgery, even observation and regularly countercheck. Whether or not TNT can maximize the proportion of patients with locally advanced disease who can be cured through non-operative management is currently being addressed in an ongoing large multicenter randomized phase II trial (47). Patients will be randomly assigned to receive FOLFOX before (inductive) or after (combined) standard chemoradiotherapy. Patients who have a complete response according to re-staging MRI and endoscopy will receive non-surgical treatment, and incomplete responders will undergo TME. The main outcome of interest is 3-year disease-free survival. Despite its advantages, the use of the TNT strategy may lead to prolonged resection times and/ or higher postoperative morbidity rates (48). At present, radical rectal surgery is still the standard chemotherapeutic option for rectal cancer, so it is important to evaluate the tumor shrinkage effect after nCRT.

The main limitation of this research is that the numbers of cases included in the 56-Gy group and the 50-Gy group were small. Also, some observation indicators have not been fully tracked; for instance, 5-year progression-free survival and total survival. are still under observation and have yet to be compared.

\section{Conclusions}

Increasing the dose of RT can significantly improve the anal preservation and $\mathrm{pCR}$ rates among patients with locally advanced rectal cancer, thus improving their quality of life. Moreover, it does not increase the rates of recurrence or adverse reactions. This strategy has high clinical value, and is worthy of further research and application.

\section{Acknowledgments}

Funding: None.

\section{Footnote}

Reporting Checklist: The authors have completed the STROBE reporting checklist. Available at https://dx.doi. org/10.21037/jgo-21-296

Data Sharing Statement: Available at https://dx.doi. org/10.21037/jgo-21-296

Conflicts of Interest: All authors have completed the ICMJE uniform disclosure form (available at https://dx.doi. org/10.21037/jgo-21-296). The authors have no conflicts of interest to declare.

Ethical Statement: The authors are accountable for all aspects of the work in ensuring that questions related to the accuracy or integrity of any part of the work are appropriately investigated and resolved. This research 
was approved by the local ethics committee of Affiliated Hospital of Qingdao University. The study was conducted in accordance with the Declaration of Helsinki (as revised in 2013). All participants in the study gave informed consent to this study and signed an informed consent form.

Open Access Statement: This is an Open Access article distributed in accordance with the Creative Commons Attribution-NonCommercial-NoDerivs 4.0 International License (CC BY-NC-ND 4.0), which permits the noncommercial replication and distribution of the article with the strict proviso that no changes or edits are made and the original work is properly cited (including links to both the formal publication through the relevant DOI and the license). See: https://creativecommons.org/licenses/by-nc-nd/4.0/.

\section{References}

1. MacFarlane JK, Ryall RD, Heald RJ. Mesorectal excision for rectal cancer. Lancet 1993;341:457-60.

2. Cedermark B, Dahlberg M, Glimelius B, et al. Improved survival with preoperative radiotherapy in resectable rectal cancer. N Engl J Med 1997;336:980-7.

3. Zaborowski A, Stakelum A, Winter DC. Systematic review of outcomes after total neoadjuvant therapy for locally advanced rectal cancer. Br J Surg 2019;106:979-87.

4. Bosset JF, Calais G, Mineur L, et al. Fluorouracilbased adjuvant chemotherapy after preoperative chemoradiotherapy in rectal cancer: long-term results of the EORTC 22921 randomised study. Lancet Oncol 2014;15:184-90.

5. Sanghera P, Wong DW, McConkey CC, et al. Chemoradiotherapy for rectal cancer: an updated analysis of factors affecting pathological response. Clin Oncol ( $R$ Coll Radiol) 2008;20:176-83.

6. Sauer R, Liersch T, Merkel S, et al. Preoperative versus postoperative chemoradiotherapy for locally advanced rectal cancer: results of the German CAO/ARO/AIO-94 randomized phase III trial after a median follow-up of 11 years. J Clin Oncol 2012;30:1926-33.

7. Lemmens V, van Steenbergen L, Janssen-Heijnen M, et al. Trends in colorectal cancer in the south of the Netherlands 1975-2007: rectal cancer survival levels with colon cancer survival. Acta Oncol 2010;49:784-96.

8. Glimelius B. Multidisciplinary treatment of patients with rectal cancer: Development during the past decades and plans for the future. Ups J Med Sci 2012;117:225-36.

9. Braendengen M, Tveit KM, Berglund A, et al. Randomized phase III study comparing preoperative radiotherapy with chemoradiotherapy in nonresectable rectal cancer. J Clin Oncol 2008;26:3687-94.

10. Tural D, Selcukbiricik F, Yildiz O, et al. Preoperative versus postoperative chemoradiotherapy in stage T3, N0 rectal cancer. Int J Clin Oncol 2014;19:889-96.

11. Roh MS, Colangelo LH, O'Connell MJ, et al. Preoperative multimodality therapy improves disease-free survival in patients with carcinoma of the rectum: NSABP R-03. J Clin Oncol 2009;27:5124-30.

12. Gérard JP, Conroy T, Bonnetain F, et al. Preoperative radiotherapy with or without concurrent fluorouracil and leucovorin in T3-4 rectal cancers: results of FFCD 9203. J Clin Oncol 2006;24:4620-5.

13. Quirke P, Steele R, Monson J, et al. Effect of the plane of surgery achieved on local recurrence in patients with operable rectal cancer: a prospective study using data from the MRC CR07 and NCIC-CTG CO16 randomised clinical trial. Lancet 2009;373:821-8.

14. Peeters KC, Marijnen CA, Nagtegaal ID, et al. The TME trial after a median follow-up of 6 years: increased local control but no survival benefit in irradiated patients with resectable rectal carcinoma. Ann Surg 2007;246:693-701.

15. Hofheinz RD, Wenz F, Post S, et al. Chemoradiotherapy with capecitabine versus fluorouracil for locally advanced rectal cancer: a randomised, multicentre, non-inferiority, phase 3 trial. Lancet Oncol 2012;13:579-88.

16. Aschele C, Cionini L, Lonardi S, et al. Primary tumor response to preoperative chemoradiation with or without oxaliplatin in locally advanced rectal cancer: pathologic results of the STAR-01 randomized phase III trial. J Clin Oncol 2011;29:2773-80.

17. Glimelius B, Tiret E, Cervantes A, et al. Rectal cancer: ESMO Clinical Practice Guidelines for diagnosis, treatment and follow-up. Ann Oncol 2013;24 Suppl 6:vi81-8.

18. Wagman R, Minsky BD, Cohen AM, et al. Sphincter preservation in rectal cancer with preoperative radiation therapy and coloanal anastomosis: long term follow-up. Int J Radiat Oncol Biol Phys 1998;42:51-7.

19. Ceelen W, Fierens K, Van Nieuwenhove Y, et al. Preoperative chemoradiation versus radiation alone for stage II and III resectable rectal cancer: a systematic review and meta-analysis. Int J Cancer 2009;124:2966-72.

20. Hwang K, Park IJ, Yu CS, et al. Impression of prognosis regarding pathologic stage after preoperative chemoradiotherapy in rectal cancer. World J Gastroenterol 2015;21:563-70. 
21. Julien LA, Thorson AG. Current neoadjuvant strategies in rectal cancer. J Surg Oncol 2010;101:321-6.

22. Yu CS, Kim TW, Kim JH, et al. Optimal time interval between capecitabine intake and radiotherapy in preoperative chemoradiation for locally advanced rectal cancer. Int J Radiat Oncol Biol Phys 2007;67:1020-6.

23. Gérard JP, Azria D, Gourgou-Bourgade S, et al. Comparison of two neoadjuvant chemoradiotherapy regimens for locally advanced rectal cancer: results of the phase III trial ACCORD 12/0405-Prodige 2. J Clin Oncol 2010;28:1638-44.

24. Rödel C, Liersch T, Becker H, et al. Preoperative chemoradiotherapy and postoperative chemotherapy with fluorouracil and oxaliplatin versus fluorouracil alone in locally advanced rectal cancer: initial results of the German CAO/ARO/AIO-04 randomised phase 3 trial. Lancet Oncol 2012;13:679-87.

25. Dias Wickramanayake P, Diehl V. Adjuvant treatment of colorectal cancer. Z Gastroenterol Verh 1988;23:51-6.

26. Francois Y, Nemoz CJ, Baulieux J, et al. Influence of the interval between preoperative radiation therapy and surgery on downstaging and on the rate of sphinctersparing surgery for rectal cancer: the Lyon R90-01 randomized trial. J Clin Oncol 1999;17:2396.

27. Tulchinsky H, Shmueli E, Figer A, et al. An interval >7 weeks between neoadjuvant therapy and surgery improves pathologic complete response and disease-free survival in patients with locally advanced rectal cancer. Ann Surg Oncol 2008;15:2661-7.

28. Kalady MF, de Campos-Lobato LF, Stocchi L, et al. Predictive factors of pathologic complete response after neoadjuvant chemoradiation for rectal cancer. Ann Surg 2009;250:582-9.

29. Evans J, Tait D, Swift I, et al. Timing of surgery following preoperative therapy in rectal cancer: the need for a prospective randomized trial? Dis Colon Rectum 2011;54:1251-9.

30. Wolthuis AM, Penninckx F, Haustermans K, et al. Impact of interval between neoadjuvant chemoradiotherapy and TME for locally advanced rectal cancer on pathologic response and oncologic outcome. Ann Surg Oncol 2012;19:2833-41.

31. Lefevre JH, Mineur L, Kotti S, et al. Effect of interval (7 or 11 weeks) between neoadjuvant radiochemotherapy and surgery on complete pathologic response in rectal cancer: a multicenter, randomized, controlled trial (GRECCAR-6). J Clin Oncol 2016;34:3773-80.

32. São Julião GP, Habr-Gama A, Vailati BB, et al. New strategies in rectal cancer. Surg Clin North Am 2017;97:587-604.

33. Habr-Gama A, Perez RO, Nadalin W, et al. Operative versus nonoperative treatment for stage 0 distal rectal cancer following chemoradiation therapy: long-term results. Ann Surg 2004;240:711-7; discussion 717-8.

34. Appelt AL, Ploen J, Harling H, et al. High-dose chemoradiotherapy and watchful waiting for distal rectal cancer: a prospective observational study. Lancet Oncol 2015;16:919-27.

35. Vaccaro CA, Yazyi FJ, Ojra Quintana G, et al. Locally advanced rectal cancer: Preliminary results of rectal preservation after neoadjuvant chemoradiotherapy. Cir Esp 2016;94:274-9.

36. Smith RK, Fry RD, Mahmoud NN, et al. Surveillance after neoadjuvant therapy in advanced rectal cancer with complete clinical response can have comparable outcomes to total mesorectal excision. Int J Colorectal Dis 2015;30:769-74.

37. Dalton RS, Velineni R, Osborne ME, et al. A single-centre experience of chemoradiotherapy for rectal cancer: is there potential for nonoperative management? Colorectal Dis 2012;14:567-71.

38. Araujo RO, Valadao M, Borges D, et al. Nonoperative management of rectal cancer after chemoradiation opposed to resection after complete clinical response. A comparative study. Eur J Surg Oncol 2015;41:1456-63.

39. Renehan AG, Malcomson L, Emsley R, et al. Watchand-wait approach versus surgical resection after chemoradiotherapy for patients with rectal cancer (the OnCoRe project): a propensity-score matched cohort analysis. Lancet Oncol 2016;17:174-83.

40. Habr-Gama A, Gama-Rodrigues J, São Julião GP, et al. Local recurrence after complete clinical response and watch and wait in rectal cancer after neoadjuvant chemoradiation: impact of salvage therapy on local disease control. Int J Radiat Oncol Biol Phys 2014;88:822-8.

41. Habr-Gama A, Perez RO, Proscurshim I, et al. Interval between surgery and neoadjuvant chemoradiation therapy for distal rectal cancer: does delayed surgery have an impact on outcome? Int J Radiat Oncol Biol Phys 2008;71:1181-8.

42. Habr-Gama A, Sabbaga J, Gama-Rodrigues J, et al. Watch and wait approach following extended neoadjuvant chemoradiation for distal rectal cancer: are we getting closer to anal cancer management? Dis Colon Rectum 2013;56:1109-17.

43. Garcia-Aguilar J, Chow OS, Smith DD, et al. Effect of 
adding mFOLFOX6 after neoadjuvant chemoradiation in locally advanced rectal cancer: a multicentre, phase 2 trial. Lancet Oncol 2015;16:957-66.

44. Perez RO. Complete clinical response in rectal cancer: a turning tide. Lancet Oncol 2016;17:125-6.

45. Maas M, Nelemans PJ, Valentini V, et al. Long-term outcome in patients with a pathological complete response after chemoradiation for rectal cancer: a pooled analysis of individual patient data. Lancet Oncol 2010;11:835-44.

46. Martin ST, Heneghan HM, Winter DC. Systematic review and meta-analysis of outcomes following pathological complete response to neoadjuvant chemoradiotherapy for rectal cancer. Br J Surg 2012;99:918-28.

Cite this article as: Zhang Y, Jiang P, Zhu H, Dong B, Zhai H, Chen $Z$. The efficacy and safety of different radiotherapy doses in neoadjuvant chemoradiotherapy for locally advanced rectal cancer. J Gastrointest Oncol 2021;12(4):1531-1542. doi: 10.21037/ jgo-21-296
47. Smith JJ, Chow OS, Gollub MJ, et al. Organ Preservation in rectal adenocarcinoma: a phase II randomized controlled trial evaluating 3-year disease-free survival in patients with locally advanced rectal cancer treated with chemoradiation plus induction or consolidation chemotherapy, and total mesorectal excision or nonoperative management. BMC Cancer 2015;15:767.

48. Ludmir EB, Palta M, Willett CG, et al. Total neoadjuvant therapy for rectal cancer: An emerging option. Cancer 2017;123:1497-506.

(English Language Editor: J. Reynolds) 